\title{
Using Protein Synthesis Inhibitors to Establish the Phylogenetic Relationships of the Sulfolobales Order
}

\author{
José L. Sanz, ${ }^{1}$ Gertrud Huber, ${ }^{2}$ Harald Huber, ${ }^{2}$ Ricardo Amils ${ }^{1}$ \\ ' Centro de Biología Molecular, Universidad Autónoma de Madrid, Cantoblanco, Madrid 28049, Spain \\ ${ }^{2}$ Lehrstuhl für Mikrobiologie der Universität Regensburg, 8400 Regensburg, Germany
}

Received: 9 December 1993 / Accepted: 3 May 1994

\begin{abstract}
The sensitivity of the cell-free protein synthesis systems from Acidanus brierleyi, Acidianus infernus, and Metallosphaera sedula, members of the archaeal order Sulfolobales, to 40 antibiotics with different specificities has been studied. The sensitivity patterns were compared to those of Sulfolobus solfataricus and other archaeal, bacterial, and eukaryotic systems. The comparative analysis shows that ribosomes from the sulfolobales are the most refractory to inhibitors of protein synthesis described so far. The sensitivity results have been used to ascertain in phylogenetic relationships among the members of the order Sulfolobales. The evolutionary significance of these results are analyzed in the context of the phylogenetic position of this group of extreme thermophilic microorganisms.
\end{abstract}

Key words: Sulfolobales - Protein synthesis - Antibiotics - Ribosomal function

\section{Introduction}

According to Vazquez (1979), protein synthesis inhibitors have been classified into three groups: (1) specific inhibitors of bacterial ribosomes, (2) specific inhibitors of eukaryotic ribosomes, and (3) inhibitors which interfere with both bacterial and eukaryotic ribosomes (universal inhibitors). Since the discovery of archaeal microorganisms, studies have shown that the ribosomes from

Correspondence to: R. Amils members of this domain exhibit a heterogeneous sensitivity to protein synthesis inhibitors (Hilpert et al. 1981; Böck et al. 1993; Hummel et al. 1985; Cammarano et al. 1985; Sanz et al. 1987, 1991; Altamura et al. 1988).

The phylogenetic merit of comparative ribosomal functional studies using protein synthesis inhibitors has received much study; the clusters obtained are similar to the phylogenetic trees generated with $16 \mathrm{~S}$ rRNA sequences, validating their phylogenetic significance (Amils and Sanz 1986; Oliver et al. 1987; Amils et al. 1989, 1990).

Recent studies have led to the suggestion that hyperthermophilic microorganisms are good candidates to be considered primitive life forms. Many hyperthermophilic microorganisms are found at the base of rooted phylogenetic trees, implying that the common ancestor of extant life exhibited this characteristic (Stetter 1993; Woese and Pace 1993).

The sensitivity patterns of cell-free protein synthesis systems from three extreme thermophilic archaea belonging to the Sulfolobales order, A. brierleyi, A. infernus, and $M$. sedula, to 40 ribosomal inhibitors are compared to those of other archaeal, bacterial, and eukaryotic systems. The remarkable insensitivity exhibited by the hyperthermophilic archaeal ribosomes to functional inhibitors is analyzed in the context of evolutionary relationships.

\section{Materials and Methods}

Strains and Culture Conditions. Acidianus brierleyi DSM 1651 and Acidianus infernus DSM 3191 were grown according to Segerer et al. 
(1986). Metallosphaera sedula DSM 5348 was grown as described (Huber et al. 1989).

Poly(U)-Directed Polyphenylalanine Synthesis. Cell-free systems were prepared following the procedure of Cammarano et al. (1985), and polyphenylalanine synthesis directed by poly(U) was carried out according to the procedure described for Sulfolobus solfataricus (Cammarano et al. 1985) with the following modifications: (1) the $\mathrm{NH}_{4} \mathrm{Cl}$ concentration for $M$. sedula was $25 \mathrm{mM}$ and (2) the incubation temperature for the A. brierleyi and $M$. sedula systems was $65^{\circ} \mathrm{C}$. Soluble factors (S-100 fraction) from S. solfataricus were used in all incubations due to its high polymerization efficiency and to facilitate comparative studies.

Statistical Methods. Statistical analysis was according Amils et al. (1989). Pairwise values were used as input to construct a quantitative matrix which was processed using the BMDP package (Dixon et al. 1983). The P2M subprogram (cluster analysis of cases) using euclidian distance was employed to find relationships among microorganisms. Simple-linkage and centroid-linkage cluster methods were used. The statistical package BIOSYS-1 (Swofford and Selander 1981) was used to obtain phylogenetic trees following Wagner's method (Swofford 1981), using the UPGMA clustering method and the following coefficients: modified Rogers distance, Cavalli-Sforza and Edwards distance, chord distance, arc distance, and " $E$ " distance (Swofford and Selander 1981).

\section{Results and Discussion}

Protein synthesis cell-free systems for A. brierleyi, $A$. infernus, and $M$. sedula have been optimized in order to perform a comparative study of sensitivity to 40 elongation inhibitors with different structural, domain, and functional specificities. The results were compared with those obtained for $S$. solfataricus ribosomes, another member of the Sulfolobales order studied earlier (Cammarano et al. 1985), and other archaeal, bacterial, and eukaryotic ribosomal systems. The insensitivity of ribosomes from the Sulfolobales order to protein synthesis inhibitors is remarkable (Table 1), suggesting a strong functional correlation between the protein synthesis machinery of these extreme thermophilic archaea.

Among specific inhibitors of bacterial ribosomes (group I antibiotics) only the aminoglycoside neomycin (Fig. 1a) inhibits at low concentration. Other aminoglycosides, tobramycin and paromomycin, as well as thiostrepton and virginiamycin $M$, are partial inhibitors at high concentration $\left(\mathrm{IC}_{50}=5 \cdot 10^{-4}-10^{-3} \mathrm{M}\right)$, which may be due to secondary effects.

Among specific inhibitors of eukaryotic ribosomes (group II antibiotics) only $\alpha$-sarcin (Fig. 1b) and the related toxins mitogillin and restrictocin inhibit protein synthesis of the Sulfolobales ribosomes, although with lower efficiency than in the eukaryotic reference system.

Of the universal antibiotics, which interfere with both bacterial and eukaryotic ribosomes (group III inhibitors), only edeine inhibits all the Sulfolobales protein synthesis systems analyzed (Fig. 1c). The peptidyltransferase in- hibitors sparsomycin (Fig. 1d), puromycin, and anthelmycin are not effective in some of the systems used (Table 1). Sensitivity of $A$. brierleyi ribosomes to sparsomycin and $A$. infernus ribosomes to puromycin, with similar sensitivities to those of the bacterial and eukaryotic reference systems (Table 1), should be underlined.

In general, ribosomes from both Acidianus species show similar inhibitory patterns, with $M$. sedula being the most antibiotic-insensitive ribosomal system analyzed. In comparative analyses of antibiotic sensitivities, the extreme insensitivity of Sulfolobales ribosomes is one of the most important characteristics.

The sensitivity data obtained has been used to generate phenograms and phylogenetic clusters. The results obtained are coherent regardless of the algorithm used to analyze them. The phylogenetic tree displayed in Fig. 2 shows that $S$. solfataricus and $M$. sedula form one cluster. A second cluster is formed with both Acidianus species, although with a low similarity coefficient. The use of sensitivity data from other archaeal, bacterial, and eukaryotic systems allowed the derivation of the correspondent phenograms. Figure 3 shows the strong correlation exhibited by the Sulfolobales ribosomes when they are compared against other ribosomes. The phenograms obtained agree with the phylogenetic clusters generated using 16S rRNA sequence analysis (Woese et al. 1990).

Although it can be argued that our conclusions are based on negative characteristics, we note that insensitivity toward ribosomal inhibitors in protein synthesis cell-free systems has strong phylogenetic value, e.g., the absolute insensitivity of all the eukaryotic ribosomes to specific bacterial inhibitors and the converse insensitivity of bacterial ribosomes to specific eukaryotic inhibitors (Table 1). Many have specific insensitivity mechanisms, such as the sensitivity of eukaryal and archaeal ribosomes to streptomycin, which has been correlated to a change of $C$ for $U$ in 16/18S rRNA (base 912 in the $E$. coli system) (Montandon et al. 1986); the insensitivity of bacterial and archaeal ribosomes to erythromycin, which has been correlated to an A-to-G change ( $E$. coli position 2058 ) in the otherwise-conserved region of domain $\mathrm{V}$ of 23/28S rRNA associated with peptidyltransferase activity (Sor and Fukuhara 1984); and the differing sensitivity of archaeal and eukaryal vs bacterial ribosomes to $\alpha$-sarcin, which correlates to an A-to-C change ( $E$. coli residue $2666)$ in an extremely conserved region close to the $3^{\prime}$ end of 23/28S rRNA (Wool 1984; Amils and Sanz 1986; Casquero and Amils, unpublished).

We also note that the insensitivity of the Sulfolobales ribosomes is not related to the ecological conditions under which these hyperthermophilic archaea grow. Appropriate controls performed with thermophilic and hyperthermophilic bacteria show that their ribosomes have a sensitivity pattern similar to the correspondent mesophilic bacteria (Cammarano et al. 1985), which strongly suggests that ribosomal insensitivity of hyperthermo- 
Table 1. Inhibition of protein synthesis in Sulfolobales ribosomes produced by different antibiotics ${ }^{\mathrm{a}}$

\begin{tabular}{|c|c|c|c|c|c|c|c|}
\hline Antibiotics & A. brier. & A. infer. & M. sedula & S. solfa. & E. coli & B. stear. & S. cerev. \\
\hline \multicolumn{8}{|l|}{ Group I } \\
\hline Althiomycin & - & - & - & - & ++ & + & - \\
\hline Carbomycin A & - & - & - & - & + & ++ & - \\
\hline Gentamicin & - & - & - & - & + & + & \pm \\
\hline Griseoviridin & - & - & - & - & + & + & - \\
\hline Kanamycin & \pm & \pm & - & - & H & + & \pm \\
\hline Neamine & + & - & - & \pm & H & + & \pm \\
\hline Neomycin & + & \pm & \pm & + & H & + & \pm \\
\hline Paromomycin & \pm & \pm & - & \pm & $H$ & H & \pm \\
\hline Ribostamycin & - & - & - & - & + & ++ & - \\
\hline Streptomycin & - & \pm & \pm & - & + & + & - \\
\hline Thiostrepton & \pm & \pm & \pm & \pm & + & + & - \\
\hline Tylosin & - & - & - & - & + & + & - \\
\hline Tobramycin & \pm & \pm & \pm & \pm & $H$ & + & \pm \\
\hline Viomycin & \pm & - & - & - & + & + & - \\
\hline Virginiamycin $\mathbf{M}$ & \pm & \pm & \pm & - & ++ & + & - \\
\hline \multicolumn{8}{|l|}{ Group II } \\
\hline Anisomycin & - & \pm & - & - & - & - & $H$ \\
\hline Bruceantin & - & - & - & - & - & - & $H$ \\
\hline Cycloheximide & - & - & - & - & - & - & + \\
\hline Cryptopleurine & - & - & - & - & - & - & + \\
\hline Heamanthamine & - & - & - & - & - & - & + \\
\hline Harringtonine & - & - & - & - & - & - & $H$ \\
\hline Mitogillin & \pm & - & \pm & - & \pm & \pm & $H$ \\
\hline Narciclasine & - & - & - & - & - & - & ++ \\
\hline Pretazetine & - & \pm & - & - & - & - & ++ \\
\hline Restrictocine & \pm & - & \pm & - & \pm & \pm & + \\
\hline$\alpha-S a r c i n$ & \pm & \pm & \pm & \pm & \pm & - & ++ \\
\hline Streptimidone & - & \pm & - & - & - & - & + \\
\hline Streptovitacin & - & \pm & - & - & - & - & + \\
\hline Toxin T2 & - & \pm & - & - & - & - & + \\
\hline Tubulosine & - & \pm & - & - & - & - & + \\
\hline Tylophorine & - & \pm & - & - & - & - & $H$ \\
\hline \multicolumn{8}{|l|}{ Group III } \\
\hline Amicetin & - & - & - & - & H & + & \pm \\
\hline Anthelmycin & + & + & - & - & $H$ & + & + \\
\hline Blasticidin S & \pm & \pm & - & - & + & \pm & $H$ \\
\hline Edein & + & + & \pm & + & + & + & + \\
\hline Fusidic acid & - & - & - & - & + & + & $H$ \\
\hline Hygromycin B & \pm & \pm & - & - & ++ & + & + \\
\hline Puromycin & \pm & + & - & - & ++ & + & + \\
\hline Sparsomycin & $H$ & \pm & - & + & ++ & $H$ & ++ \\
\hline Tetracycline & \pm & + & - & - & + & $H$ & + \\
\hline
\end{tabular}

${ }^{a}$ Group I: bacterial-targeted antibiotics; group II: eukaryotic-targeted antibiotics; and group III: antibiotics affecting both bacteria and eukarya. Symbols: ++: similar inhibitory profile to that of the reference system; +: one order of magnitude less active than the reference system; \pm : two orders of magnitude less active than the reference system;

philic archaea is related to their phylogenetic characteristics rather than to the conditions in which the microorganisms develop.

The remarkable insensitivity of Sulfolobales ribosomes to translation inhibitors poses an interesting question regarding the evolution of ribosomal functions. The Crenarchaeota are good candidates for the most ancestral phenotype because of (1) their hyperthermophilic character (Woese and Pace 1993); (2) their relatively small genome size (Noll 1989; Yamagishi and Oshima 1990);
-: no activity at the maximun concentration assayed. A. brier.: Acidianus brierleyi; A. infer.: Acidianus infernus; $M$. sedula: Metallosphaera sedula; $S$. solfa.: Sulfolobus solfataricus; $E$. coli: Escherichia coli; B. stear.: Bacillus stearothermophilus; S. cerev.: Saccharomyces cerevisiae

(3) the ability of their tRNAs to replace endogenous tRNAs from protein cell-free systems from the three domains (universal adapters) (Amils et al., unpublished results); and (4) their energy-generating mechanisms $\left(S^{0}\right.$ chemolithoautotrophy) (Wächterhäuser 1990; Stetter 1993). The insensitivity of their translational apparatus to protein synthesis inhibitors suggests a primitive ribosome, lacking binding sites for most known antibiotics. The binding sites would presumably have accumulated at later stages of evolution. Maximum parsimony 

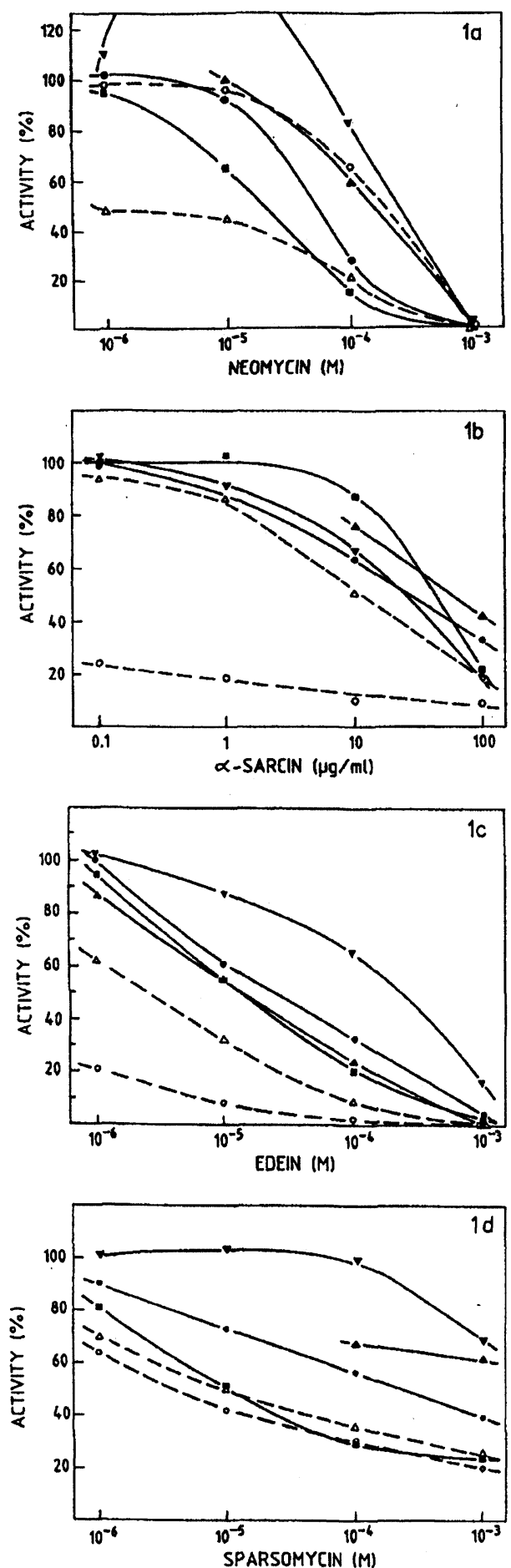

Fig. 1. Effect of selected inhibitors on phenylalanine incorporation directed by poly(U) in cell-free systems of the Sulfolobales. The protein synthesis conditions for the different systems are those described in the Materials and Methods section. E. coli and S. cerevisiae were used as reference systems. Symbols: $\boldsymbol{O}$. S. solfataricus; $\boldsymbol{\square}, A$. brierleyi; $\boldsymbol{\Lambda}$, $A$. infernus; $\nabla, M$. sedula; $\triangle, E$. coli; $\bigcirc, S$. cerevisiae.

analysis of the sensitivity data supports this model, assigning to the progenote only partial sensitivity to some inhibitors of the group III antibiotics (universal inhibitors). A careful analysis of the intersection of structural

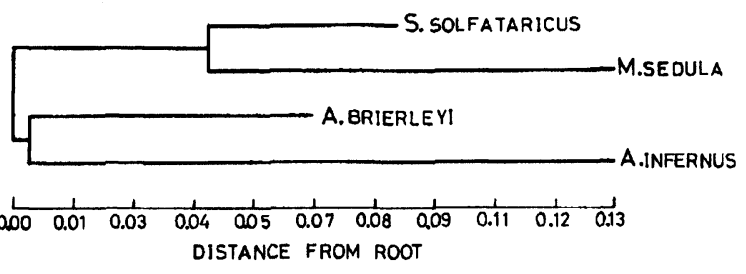

Fig. 2. Phylogenetic tree of the Sulfolobales group of archaea obtained by Wagner parsimony analysis of their ribosomal inhibition data. The functional data used are those of Table 1. The BIOSYS-1 program (UPGMA clustering method and modified Rogers distance as coefficient) was used for the statistical analysis.

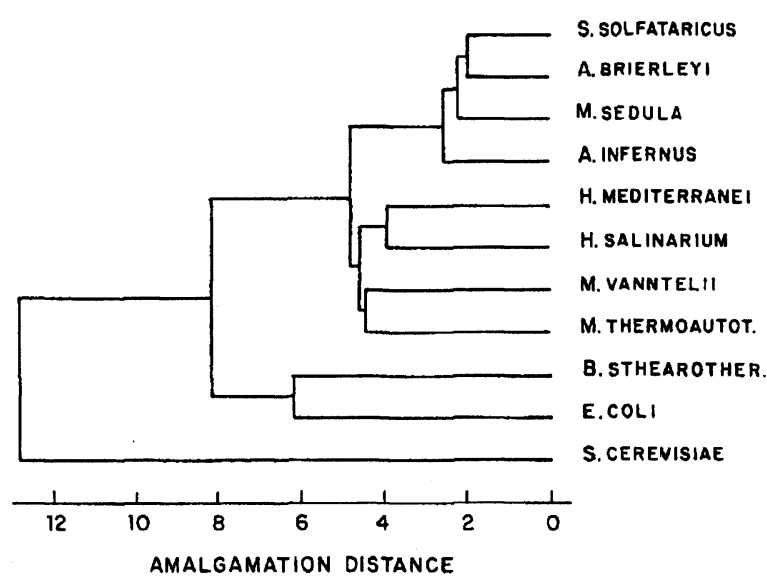

Fig. 3. Phenetic relationships among archaea, bacteria, and eukaryotes using antibiotic sensitivity data. The functional data used are those from the inhibition curves of the present work and from $H$. salinarium and $H$. mediterranei (Sanz et al. 1993); M. thermoautotrophicum and $M$. vannielii (Sanz, Hummel, Böck, and Amils, unpublished results); and E. coli, B. stearothermophilis, and S. cerevisiae (Cammarano et al., 1985). The inhibition curves were quantified using the algorithm described in Amils et al. (1989). The BMDP program (P2M subprogram and the single linkage clustering methods) was used for the statistical analysis.

and functional data and the use of powerful dissection techniques like footprinting and site-directed mutagenesis using different ribosomes from different phylogenetic domains should provide insight into this fundamental paradigm of evolutionary biology.

\section{References}

Altamura S, Sanz JL, Amils R, Cammarano P, Londei P (1988) The antibiotic sensitivity spectra of ribosomes from the Thermoproteales: phylogenetic depth and distribution of antibiotic binding sites. System Appl Microbiol 10:231-240

Amils R, Sanz JL (1986) Inhibitors of protein synthesis as phylogenetic markers. In: Hardesty B, Kramer G (ed) Structure, function and genetic of ribosomes. Springer Series in Molecular Biology, Springer Verlag, New York, pp 605-620

Amils R, Ramírez L, Sanz JL, Marín I, Pisabarro AG, Ureña D (1989) The use of functional analysis of the ribosome as a tool to determine archaebacterial phylogeny. Can J Microbiol 35:141-147

Amils R, Ramírez L, Sanz JL, Marín I, Pisabarro AG, Sánchez M, Ureña D (1990) Phylogeny of antibiotic action. In: Hill WE, Dahl- 
berg A, Garret RA, Moore PB, Schlessinger D, Warner JR (ed) The ribosome. American Society of Microbiology, Washington DC, pp 645-654

Böck A, Bär U, Schmid G, Hummel H (1983) Amino-glycoside sensitivity of ribosomes from the archaebacterium Mathanococcus vannielii: structure-activity relationships. FEMS Microbiol Lett 20: $435-438$

Cammarano P, Teichner A, Londei P, Acca M, Nicolaus B, Sanz JL, Amils R (1985) Insensitivity of archaebacterial ribosomes to protein synthesis inhibitors. Evolutionary implications. EMBO J 4: 811-816

Dixon WJ, Brown MB, Engelman L, Frane JW, Hill MA, Jennrich RI, Toporek JD (1983) BMDP statistical software. University of California Press, Berkeley

Hilper R, Winter J, Hammes W, Kandler O (1981) The sensitivity of archaebacteria to antibiotics. Zentralbl Bakterol Hyg, I Abt Orig C 2:11-20

Huber G, Spinnler C, Gambacorta A, Stetter KO (1989) Metallosphaera sedula gen. and sp. nov. represents a new genus of aerobic, metal-mobilizing, thermoacidophilic archaebacteria. System Appl Microbiol 12:38-47

Hummel H, Bär U, Heller G, Böck A (1985) Antibiotic sensitivity patter of in vitro polypeptide synthesis systems from Methanosarcina barkeri and Methanospirillum hungatei. J Biol Chem 260: 529-533

Montandon PE, Wagner R, Stutz E (1986) E. coli ribosomes with a $\mathrm{C}_{912}$ to $\mathrm{U}$ base change in the $16 \mathrm{~S}$ rRNA are streptomycin resistant. EMBO J 5:3705-3708

Noll KM (1989) Chromosome map of the thermophilic archaebacterium Thermococcus celer. J Bacteriol 171:6720-6725

Oliver JL, Sanz JL, Amils R, Marin A (1987) Inferring the phylogeny of archaebacteria: the use of ribosomal sensitivity to protein synthesis inhibitors. J Mol Evol 24:281-288

Sanz JL, Altamura S, Mazziotti I, Amils R, Cammarano P (1987) Unique antibiotic sensitivity of an in vitro polypeptide synthesis system from the archaebacterium Thermoplasma acidophilum. Phylogenetic implications. Mol Gen Genet 207:385-394
Sanz JL, Marín I, Ureña D, Amils R (1991) Functional analysis of seven ribosomal systems from extremely halophilic archaea. Can J Microbiol 38:311-317

Segerer A, Neuner A, Kristjansson JK, Stetter KO (1986) Acidianus infernus gen. nov., sp. nov., and Acidianus brierleyi comb. nov.: facultatively aerobic, extremely acidophilic thermophilic sulfurmetabolizing archaebacteria. Int J System Bacteriol 36:559-564

Sor F, Fukuhara H (1984) Erythromycin and spiramycin resistance mutations in the mitochondrial gene coding for the large rRNA gene in yeast. Nucleic Acids Res 12:8313-8318

Stetter KO (1993) The lesson of archaebacteria. In: Benstson S (ed) Early life on earth: Nobel symposium No 84. Columbia University Press, New York (in press)

Swofford DL (1981) On the utility of the distance Wagner procedure. In: Funk VA, Brooks DR (ed) Advances in cladistics. New York Botanical Garden, New York, pp 25-43

Swofford DL, Selander RB (1981) BIOSYS-1: a FORTRAN program for the comprehensive analysis of electroforetic data in population genetics and systematic. J Hered 72:281-283

Vázquez D (1979) Inhibitors of protein biosynthesis. In: Kleinzeller A, Springer GF, Wittmann HG (ed) Molecular biology, biochemistry and biophysics 30 . Springer Verlag, Berlin

Wächterhäuser G (1990) The case for the chemoautotrophic origins of life in an iron-sulfur world. Orig Life 20:173

Woese CR, Kandler O, Wheelis ML (1990) Towards a natural system of organisms: proposal for the domains Archae, Bacteria, and Eucarya. Proc Natl Acad Sci USA 87:4576-4579

Woese CR, Pace NR (1993) Probing RNA Structure, Function, and History of Comparative Analysis. In: Gesteland RF, Atkins JF (ed) The RNA world. Cold Spring Harbor Laboratory Press, Cold Spring Harbor, NY, pp 91-135

Wool IG (1984) The mechanism of action of the cytotoxic nuclease $\alpha$-sarcin and its use to analyse ribosome structure. Trends Biochem Sci 9:14-17

Yamagishi A, Oshima T (1990) Circular chromosomal DNA in the sulfur-dependent archaebacterium Sulfolobus acidocaldarius. Nucleic Acids Res 18:1133-1136 